\title{
CRITICAL SUBMANIFOLDS OF DIFFERENTIABLE MAPPINGS
}

\section{BY SAMIR A. KHABBAZ AND EVERETT PITCHER}

Communicated by E. Pitcher, August 1, 1966

1. The problems and definitions. There is a general type of problem which contains critical point theory at one extreme, and immersion theory at another. The problems of interest to us lie between these two theories. A glance into their nature is afforded by a simple example to be given following some definitions. Let $N^{n}$ and $M^{m}$ denote two differentiable manifolds-with-boundary (perhaps empty) of dimensions $n$ and $m$ respectively, and let $f: N \rightarrow M$ be a continuous function with sufficient differentiability at any stage to allow the discussion to proceed. The deficiency of $f$ at a point $x$ of $N$ is defined by (minimum $(n, m)$-rank $f$ at $x$ ). Then $x$ is said to be an ordinary point of $f$ if $f$ has deficiency zero at $x$; otherwise $x$ is called a critical point of $f$. If each point of $N$ is an ordinary point of $f$, we shall simply say $f$ is ordinary. Note that if $f$ is ordinary and $n \leqq m$ then $f$ is just an immersion, while if $n \geqq m$ then (in terms of suitable coordinate systems) $f$ is locally a projection.

To proceed with the example, let $S^{n}$ denote the unit sphere in the $(n+1)$-dimensional euclidean space $R^{n+1}$, and consider the map $f: S^{n} \rightarrow R^{r}$ (induced in this instance by the natural projection $R^{n+1}$ $\left.\rightarrow R^{r}\right), r \leqq n$. Then we observe that: (a) the set of critical points of $f$ is confined to the submanifold $S^{r-1}$ of $S^{n}$; (b) $f \mid\left(S^{n}-S^{r-1}\right)$, the restriction of $f$ to the complement of $S^{r-1}$ in $S^{n}$, and $f \mid S^{r-1}$ are ordinary; and (c) there exists a map $g: R^{r} \rightarrow R$ (here the natural projection $R^{r} \rightarrow R^{1}$ ) such that $g f$ and $(g f) \mid S^{r-1}$ are Morse functions having the same number of critical points. Now if one attempts to replace $S^{n}$ in the above by a compact manifold $N^{n}$ and $S^{r-1}$ by a submanifold $K$ of $N$, one is immediately faced with the questions of which pairs $(N, K)$ are admissible and what types of singularities to expect? Should it be possible to find an $f: N \rightarrow R^{r}$ satisfying the modified (a) and (b), $N-K$ must for instance admit $r$ linearly independent vector fields and $K$ must be immersible in $R^{r}$; while the addition of (c) would require that the Euler characteristics of $K$ and $N$ be congruent modulo two, since the number of critical points of a Morse function defined on a compact manifold is congruent modulo two to the Euler characteristic. These are some aspects of problems which we consider.

In this paper we give a condition of a local nature for the set of critical points of $f$ in the deficiency 1 case to be (not just to be con- 
fined to) a submanifold of $N$, and conclude with a section concerning the effect on the structure of $N$ of the existence of a function $f: N \rightarrow R^{r}$ subject to conditions weaker than (a) and (b) above. The results in this section depend largely on the behavior of $f \mid(N-K)$ and $f \mid K$, and make no essential use of the crucial behavior of $f$ in a neighborhood of $K$. We shall take up this latter question in a subsequent publication.

We conclude this section with a historical note. The classical critical point theory of Morse is concerned with the case $r=1$ and $M=R$.

The remaining case for $r=1$, namely $f: N^{n} \rightarrow S^{1}$, has been discussed for a compact manifold $N$ by one of the writers [3]. The attack consists of lifting $f$, with greatest economy, to a covering map $g$ in the diagram

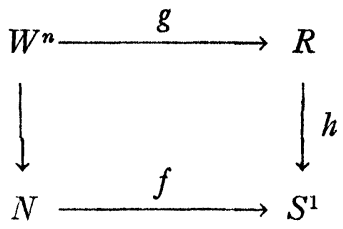

The function $h g$ is invariant under the appropriate factor group of $\pi_{1}(N)$ and ordinary critical point theory can be applied to $g$ on a suitable fundamental domain.

The case $r=n$, which will be seen to be of special significance, has been treated by Tucker [4]. Fiberings with singularities have been discussed in various terms, for instance [2]. There is also a general spectral theory of maps by Fary [1].

2. Deficiency 1. An example of deficiency 1 is the map $\left(x^{1}, \cdots, x^{n}\right)$ $\rightarrow\left(Q(x), x^{2}, \cdots, x^{r}\right)$, where $Q$ is a nondegenerate quadratic form and $r \leqq n$. If $Q$ is a definite form, this is intuitively a "fold" about the plane $Q_{x^{1}}=0, Q_{x^{r+1}}=0, \cdots, Q_{x^{n}}=0$. The term "fold" is most intuitive when $r=n$.

THEOREM 1. If $x_{0}$ is a critical point of $f: R^{n} \rightarrow R^{r}, n \geqq r$, of deficiency 1 and if the critical point of $F=\lambda_{i} f^{i}$, with multipliers $\lambda \neq 0$, at $x_{0}$ is nondegenerate, then the critical points of $f$ near $x_{0}$ form a manifold of dimension $r-1$.

Proof. A change in coordinates in $R^{n}$ and $R^{r}$ reduces the problem to the case in which $x_{0}=0, f_{x^{i}}^{7}(0)=0,\left|f_{x^{p} x^{q}}^{r}(0)\right| \neq 0$ with $p, q=1,2$, $\cdots, r-1$, and there is no solution except $(c)=(0)$ for the system $c_{n} f_{x^{j}}^{p}(0)=0$. Then the equations 


$$
\begin{array}{r}
v_{p} f_{x^{j}}^{p}+u f_{x^{j}}^{r}=0, \\
v_{p} v_{p}+u^{2}=1
\end{array}
$$

admit solutions $x^{j}=\phi^{j}(v)$, (and also, for reference, $u=\psi(v)$ ), by virtue of the implicit function theorem, with $(x, u, v)$ near $(0,1,0)$. Further the solution defines a manifold as required. To see this, note that $v_{p} f_{x^{i}}^{p}(\phi)+\psi f_{x^{i}}^{r}(\phi)=0$ so that

$$
f_{x^{j}}^{q}(\phi)+v_{n} f_{x^{j} x^{j h}}^{p} \phi_{v_{q}}^{h}+\psi_{v_{h}} f_{x^{j}}^{r}(\phi)+\psi f_{x_{i} x^{h} \phi_{v_{q}}^{r}}^{r}=0 .
$$

At the initial solution $f_{x^{j}}^{q}(0)+f_{x^{j} x^{h}}^{r}(0) \phi_{v_{\alpha}}^{h}(0)=0$. If there were numbers $(c) \neq(0)$ such that $\phi_{v_{q}}^{h}(0) c_{q}=0$, it would follow that $c_{q} f_{x^{i}}^{q}(0)=0$, contrary to hypothesis.

3. Relationship to Stiefel-Whitney classes. The following conventions will be used throughout. Let $N$ denote an $n$-dimensional compact connected differentiable manifold, let $K$ denote a compact $k$ dimensional differentiable submanifold-with-boundary of $N$, and let $N-K$ denote the complement of $K$ in $N$. Unless the contrary is implied, we shall use the singular cohomology theory with coefficient domain $Z_{2}$. If $V$ is an $n$-plane bundle over $X$ and $Y$ is a subspace of $X$ we shall denote by $V \mid Y$ the restriction of $V$ to $Y$. As usual $w_{i}(V)$ and $\bar{w}_{i}(V)$ will respectively denote the $i$ th Stiefel-Whitney class and the dual $i$ th Stiefel-Whitney class of $V$; while $w(V)$ and $\bar{w}(V)$ will denote the corresponding total classes. If $M$ is a differentiable manifold with boundary, $\tau(M)$ will denote the tangent bundle of $M$; and $w_{i}(M)$ will denote $w_{i}(\tau(M))$ the $i$ th Stiefel class of $M$, etc. Finally $P^{m}$ will denote the real $m$-dimensional projective space, and $R^{r}$ will denote the $r$-dimensional euclidean space. We will always assume that $n \geqq r$.

For the purposes of the following theorem let $L$ be a disjoint union of compact submanifolds-with-boundary of $N$ having maximum dimension $k$.

TheOREM 2. With $L$ and $N$ as above, assume that there exists an ordinary mapping $f: N-L \rightarrow R^{r}$. Then $w_{j}(N)=0$ for all $j$ satisfying $n-r<j<n-k$.

Proof. The fact that $f$ is ordinary implies that $\tau(N-L)$ is the Whitney sum of an $(n-r)$-plane bundle and a trivial $r$-plane bundle. Hence $w_{t}(N-L)=0$ for $t>n-r$. Moreover it follows from Poincaré duality that $H^{t}(N, N-L)=0$ for $t<n-k$, so that $i^{*}: H^{t}(N)$ $\rightarrow H^{t}(N-L)$ is a monomorphism in this range. The proposition follows since $w_{j}(N-L)=i^{*}\left(w_{j}(N)\right)$. 
Corollary. Suppose that $n, j, k$ and $r$ are integers satisfying $n-r$ $<j<n-k$ and that the binomial coefficient $C_{n+1, j}$ is odd. (For example for $n=2^{a}-2$ and $k \leqq r-2$ any $j$ strictly between $n-r$ and $n-k$ will $d o$ ). Then there exists no ordinary mapping $f:\left(P^{n}-L\right) \rightarrow R^{r}$.

For the next theorem recall that if $K$ is immersible in $R r$, then $\bar{w}_{i}(K)=0$ for $i>r-k$.

Theorem 3. Let $K$ be a compact (not necessarily connected) $k$-dimensional submanifold-with-boundary of $N$, and assume that:

(1) $w_{1}(N)=\cdots=w_{n-r}(N)=0$ if $n>r$,

(2) $w_{n-k}(N)=0$ if $k<n$,

(3) $\bar{w}_{i}(K)=0$ for all positive $i>n-k$,

(4) there exists an ordinary mapping $f:(N-K) \rightarrow R$.

Then the characteristic ring of $N$ is trivial $\left(\right.$ i.e. $w_{s}(N) \cdot w_{t}(N)=0$ for all $s>0$ and $t>0$ ).

Proof. Fixing a Riemannian metric on $N$, let $W$ be the normal bundle of $K$ in $N$, and write $\tau(N) \mid K$ as the Whitney sum $\tau(K) \oplus W$. Then (1), (2), (4), Theorem 2, and the naturality of the $w_{i}$ 's imply that $w(W) w(K)=1+$ terms of degree greater than $n-k$. Since $W$ is an $(n-k)$-plane bundle this implies in view of (3) that $\bar{w}(K)=w(W)$ and hence $w(\tau(N) \mid K)=1$. Thus $i^{*}\left(w_{i}(N)\right)=0$ for $i \geqq 1$, where $i^{*}: H^{i}(N)$ $\rightarrow H^{i}(K)$ is induced by inclusion. Let $T$ be a small compact tubular neighbourhood of $K$ in $N$, (if $k=n$ let $T=K$ ), and let $C$ be the closure of $N-T$. Now suppose integers $s$ and $t$ exist which contradict the conclusion of the theorem. Since the inclusion $K \rightarrow T$ is a homotopy equivalence we conclude from the last equation that there exists an element $a_{s}$ in $H^{s}(N, T)$ mapping onto $w_{s}(N)$ under the map $H^{s}(N, T)$ $\rightarrow H^{s}(N)$ induced by inclusion. Next, as in the proof of Theorem 2 , the fact that $f \mid C$, more correctly $f \mid(C \cap(N-K))$, is ordinary implies that $w_{i}(C)=0$ for $i>n-r$; and since $\tau(C)=\tau(N) \mid C$ it follows from (1) that $w(C)=1$. Again there is an element $a_{t}$ in $H^{t}(N, C)$ mapping onto $w_{t}(N)$ under the natural map $H^{t}(N, C) \rightarrow H^{t}(N)$. However $a_{s} \cdot a_{t}$ $\in H^{s+t}(N, T \cup C)=0$, which contradicts $w_{s}(N) \cdot w_{t}(N) \neq 0$ by the naturality of cup products.

Corollary. Suppose that $K$ is an $(n-1)$-dimensional compact submanifold of $P^{n}$ where $n$ is an odd integer not of the form $2^{a}-1, a$ an integer. Then there exists no differentiable mapping $f: P^{n} \rightarrow R^{n}$ such that $f \mid\left(P^{n}-K\right)$ and $f \mid K$ are ordinary.

The proof of the following theorem is similar to that of Theorem 3 and is more straightforward. Note that if a compact orientable $(r-1)$-dimensional manifold $M$ is immersible in $R^{r}$ then $w(M)=1$. 
THEOREM 4. Let $K$ be a k-dimensional compact submanifold-withboundary of $N$ and assume further that: (1) $w(K)=1$, and (2) for some integers $s$ and $t$ satisfying $s \geqq n-r+1$ and $t \geqq n-k+1$ we have $w_{s}(N)$ $\cdot w_{t}(N) \neq 0$. Then there exists no ordinary mapping $f:(N-K) \rightarrow R r$.

Corollary. Suppose $n$ has the form $2^{a}-2, a>2$; and suppose that $K$ is a compact orientable $(r-1)$-dimensional submanifold of $P^{n}$ where $2 r \geqq n+3$. Then there exists no differentiable mapping $f: P^{n} \rightarrow R^{r}$ such that $f \mid(N-K)$ and $f \mid K$ are ordinary.

\section{BIBLIOGRAPHY}

1. I. Fary, Valeurs critiques et algêbres spectrales d'une application, Ann. of Math. 63 (1956), 437-490.

2. D. Montgomery and H. Samelson, Fiberings with singularities, Duke Math. J. 13 (1946), 51-56.

3. E. Pitcher, Critical points of a map to a circle, Proc. Nat. Acad. Sci. U.S.A. 25 (1939), 428-431.

4. A. W. Tucker, Branched and folded coverings, Bull. Amer. Math. Soc. 42 (1936), 859-862.

LEHIGH UNIVERSITY 